\title{
DESDE LOS ÁMBITOS DE ENFERMERÍA, ANALIZANDO EL CUIDADO HUMANIZADO
}

\author{
FROM THE CONTEXT OF NURSING, ANALYZING \\ THE HUMANIZED CARE
}

\author{
Paula Andrea Ceballos Vásquez
}

\begin{abstract}
RESUMEN
Este artículo plantea el análisis del cuidado que es entregado por las enfermeras en los diferentes ámbitos del desarrollo profesional. Se realza el valor del cuidado humanizado para la enfermería y se visualiza que esta mirada humana es necesaria en todas las áreas de desarrollo profesional enfermera(o): la gestión, educación, investigación y asistencia. Se destaca que el cuidado humanizado es más que brindar un buen trato o sólo satisfacción usuaria, sino donde "el otro" necesita ser cuidado; de esta forma el equipo de enfermería crece junto al usuario y su familia, se identifican con ellos, otorgándoles cuidados holísticos centrados en la persona.
\end{abstract}

Palabras clave: Enfermería, cuidados, humanización en la atención, rol profesional.

\begin{abstract}
This article presents the analysis of nursing care in different contexts of professional development. Improves the humanized care value for nursing, and visualize this human approach as necessary in every area of their professional development, such as management, education, research and assistance. Emphasizes that humanized care is more than providing a good treat or users satisfaction, further on, is where "the other" needs to be cared. Therefore, the nursing team grows along the users and their families, and identify with them, giving holistic cares centered on the person.
\end{abstract}

Key words: Nursing, care, assistance of humanization, professional role.

Fecha recepción: 31/07/09 Fecha aceptación: 22/03/2010

"Ante el riesgo de deshumanización en el cuidado del paciente, a causa de la gran reestructuración administrativa de la mayoría de los sistemas del cuidado de salud en el mundo, se hace necesario el rescate del aspecto humano, espiritual y transpersonal, en la práctica clínica, administrativa, educativa y de investigación por parte de los profesionales de enfermería"(1).

Este aspecto es clave en relación a la forma actual de entregar cuidados de enfermería, especialmente en Chile, donde existe pre- ocupación por la inclusión de la gestión del cuidado en el Código Sanitario, y de otros temas que no dejan de ser relevantes para la práctica profesional en enfermería, razón por la cual se ha dejado de lado el centro del quehacer: la persona, el individuo, el usuario, el paciente, el cliente o como sea llamado el "ser humano". Es importante definir entonces el concepto de persona; según Watson es "un ser en el mundo, como una unidad de mente - cuerpo y espíritu, que experimenta y percibe conceptualmente el gestalt, es el 
locus de la existencia humana y el sujeto de cuidado de enfermería" (2). Esta persona demanda compromiso, dedicación, respeto, y es responsabilidad del profesional de enfermería el cuidarlo. Watson se extiende más allá e indica que el cuidado humanizado se debe trabajar en todas las áreas de desarrollo de la enfermería, en la administración o actualmente llamada gestión, en la asistencia, en la formación y la investigación en enfermería (3). Áreas o ámbitos que serán analizadas en este escrito.

Desde el área de la Gestión, según el artículo 113 del código sanitario chileno: "Los servicios profesionales de la enfermera comprenden la gestión del cuidado en lo relativo a promoción, mantención y restauración de la salud, la prevención de enfermedades o lesiones, y la ejecución de acciones derivadas del diagnóstico y tratamiento médico y el deber de velar por la mejor administración de los recursos de asistencia para el paciente" (4).

Sin duda fue un gran logro para la Enfermería chilena haber sido incorporada al código sanitario en el año 1997, pareciera que esto ha traído consecuencias en lo que al cuidado se refiere ya que las enfermeras(os) han centrado su quehacer en lo administrativo, dejando en segundo plano el deber de velar por la persona, aspecto que se considera en la asistencia del paciente y se relaciona estrechamente con el "cuidado humanizado". Es este aspecto el que provoca controversias: ¿Cómo podríamos cuidar a un individuo olvidando su humanidad?, pensar en el cuidado deshumanizado del que habla Watson es lo que parece más insólito, el cuidar sin empatía, sin respeto, sin el cariño con que se debe cuidar del otro, olvidando que es el centro del quehacer enfermero, tal como nos indica Álvarez: "El ejercicio profesional del equipo de salud adolece actualmente de un proceso de deshumanización en la sociedad globalizada, en las cuales prevalece un vacío espiritual y la cultura de la banalidad"(5).

La gestión es importante para organizar los cuidados con lo recursos suficientes, pero no lo es todo para la enfermería; gestionar para cuidar, no existir sólo para gestionar. Como indica, Leininger en MacFarland: "Los cuidados culturalmente congruentes son los que dejan al paciente convencido que recibió buenos cuidados, de calidad" (6).

Al observar la enfermería desde el área asistencial, algunos autores indican:"Es difícil mantener los valores humanitarios en el acto de cuidar en las instituciones públicas de salud, en donde parecen invisibilizarse los cuidados de enfermería por las labores biomédicas, pero las enfermeras(os) deben mantener aún entonces las virtudes de comunicarse con el otro, ayudarlo y sostenerlo en esta difícil etapa de su vida" (7).

Otro aspecto que aleja a la enfermería del "cuidado humanizado" es el tecnologicismo, es decir, la relevancia entregada por los profesionales de enfermería a las técnicas, a los procedimientos y a las supervisiones $\mathrm{u}$ otros aspectos relevantes e importantes de su quehacer, pero que no reemplazan la entrega y cariño hacia los pacientes, estos dos aspectos deben ser complementarios y no contrarios. Como indica Leininger en MacFarland: "Las actitudes y la práctica de los cuidados es lo único que distingue a la enfermería de las contribuciones de otras disciplinas" (6). He aquí la importancia que el cuidado tiene para esta profesión, el empaparse de ellos otorgará a esta disciplina la tan anhelada autonomía profesional.

El realizar un procedimiento con altos estándares de calidad, es otra manera de cuidar con respeto, pero según los mismos usuarios el hacerlo bien no es tan importante para ellos como hacerlo con entrega y empatía. Realizando un real encuentro con el "otro" según Levinas: "El encuentro infinito entre el mismo y el otro. Un encuentro que nace de la esencia incompleta de los seres humanos... encuentro que nutre, en el que el Mismo busca al Otro y en donde los dos tratan de completarse aun siendo seres incompletables" (8). 
Muchos estudios avalan esta afirmación e indican que los clientes valoran más los aspectos del cuidado que tienen relación con la comunicación efectiva, el afecto, y la atención que se les entrega dentro de los hospitales (7).

Además de preocuparse de los aspectos biomédicos, las enfermeras le otorgan mucha importancia a la tecnología, entre más tecnologizado sea el cuidado pareciera que "es mejor", creencia algo desafortunada si analizamos el transfondo que implica alzar la tecnología por sobre el ser humano, que siente dolor, angustia, ansiedad, sumado a una familia que tiene dudas, miedos, a la cual hay que acoger, escuchar, educar; en definitiva, "CUIDAR" (9). Este aspecto es de responsabilidad del equipo de enfermería y en este trabajo se debe integrar al resto del equipo de salud.

El trabajar con un paciente que no se comunica verbalmente, como es el paciente de una unidad crítica, no quiere decir que pierda su humanidad, es un ser en desventaja, desvalido pero no pierde su condición de persona, es por eso que enfermería debiera trabajar en conjunto con la tecnología pero no vivir para ella. El equipo de enfermería se esmera en perfeccionarse sobre manejo de ventiladores, de catéteres, monitores y falta preparación para cuidar iEstaremos equivocando el camino?, es bueno apoyarse en la tecnología, pero sin olvidar el centro del quehacer: la persona.

La humanización del cuidado requiere tiempo, recursos, buena relación intergrupal y compromiso explícito de la institución (10). Esto no quiere decir que realizar cuidado humanizado cueste más dinero, sino que se debe invertir en ello y "gestionar" los recursos de mejor manera para poder lograr la contratación de más personal, ambientar los servicios para entregar una atención personalizada al usuario y su familia, al incorporar otros aspectos importantes en la atención de éstos.

El área de la formación es otro punto im- portante a comentar en este escrito, ya que la manera de formar marcará al futuro profesional en lo que a cuidado se refiere.

Según estudios existen características que deben tener o poseer las personas que entregan los cuidados, también hay numerosos documentos que orientan a la enfermería para entregar "el cuidado humanizado" (6, $8,11)$.

El "profesional cuidador" debe poseer algunas características especiales como: capacidad de comunicación y escucha, respeto por las creencias del otro, gran dimensión de entrega, compromiso ético y calidez humana. Furegato señala que "los pacientes requieren a la enfermera siempre presente para atender su llamado, escuchar su angustia" (11). Lo que hace la diferencia entre dar atención de enfermería y el cuidado humanizado.

Estas relevantes características no están del todo desarrolladas en los profesionales enfermeros(as), entonces la pregunta es ¿qué se esta haciendo al respecto? Aquí se torna importante la formación que se les entrega a los estudiantes de enfermería en el pregrado, el sello que los impregna desde las escuelas de enfermería, explícito en los perfiles de egreso, Misión y Visión. Watson refiere: " $\mathrm{La}$ mayoría de los estudiantes reconocen en sus profesores la importancia de las actitudes del cuidado para su aprendizaje" (3).

Es por esto que las enfermeras dedicadas a la formación deben ser modelos para sus estudiantes, líderes en el cuidado humano, tanto para los usuarios como para ellos mismos. Muchas veces los estudiantes requieren también de cuidados por parte de sus profesores, requieren respeto, comunicación empática, calidez humana (9).

Hay que agregar, además, que el modelo formador está centrado en el cuidado del paciente recuperable o con posibilidades de rehabilitación, los estudiantes se preparan para "triunfar" con sus cuidados (12). En este sentido, no se forman enfermeros que cuiden la agonía, y entreguen cuidados para el bien morir del paciente; esta preparación 
no sólo requiere de profesionales del cuidado, sino también requiere de una formación filosófica y psicológica que muchas veces los estudiantes de pregrado ven como "poco relevante", priorizan técnicas, y lo biomédico, resultado de los ejemplos formadores que tienen en su vida universitaria. Así seguirán siendo enfermeras biomédicas y deficientes cuidadoras.

Con lo anterior, se puede visualizar que no sólo es importante invertir en recursos humanos en los servicios de salud, en estructurar a los hospitales como "hospitales amigo", sino también hay que trabajar en la formación de futuras enfermeras, que comprendan la importancia de los cuidados humanizados para el paciente, la mejora de la satisfacción del usuario interno como externo, y el trabajo que se debe hacer con las docentes para ser ejemplos de formación.

La investigación es un espacio algo distante para la enfermería chilena, la cual ha estado concentrada en lo asistencial y administrativo. Aunque en los últimos años, algunas enfermeras, visualizaron la importancia de la investigación para la disciplina y han sido pioneras en su desarrollo, hecho muy motivador para las nuevas generaciones. Los futuros profesionales deben tener claro que la única forma de conocer el impacto que producen los cuidados de enfermería, es a través de la investigación. Además de que el generar nuevos conocimientos entrega a la enfermería una mayor autonomía profesional. He aquí la importancia de trabajar este ámbito de la profesión desde el pregrado. Según Watson: "La teoría del caring reitera de manera continua la necesidad de buscar un nuevo conocimiento y una nueva práctica del cuidado" (3).

Otro punto a destacar en el ámbito de la investigación es la ética, la que solamente se mencionará pero que es necesaria tenerla presente. No se debe perder el respeto por las personas por lograr algo; nuevamente la formación profesional aquí es fundamental, educar sobre normas internacionales, consentimien- to informado, protección al usuario (13).

\section{CONSIDERACIONES FINALES}

- El cuidado humanizado es más que un buen trato o satisfacción usuaria, es que "el otro" necesita ser cuidado y al realizarlo el equipo de enfermería y el usuario obtienen frutos, no sólo recibe el paciente y su familia sino que también el profesional y el equipo que entrega los cuidados, se aprende y se crece junto con ellos.

- Comprendemos que esta mirada humana es útil para todas las áreas de desarrollo del profesional enfermera $(0)$, no sólo para el área asistencial. En la gestión es muy importante ya que indica la mejor forma de organizar los recursos con el fin de entregar un cuidado humano. En lo asistencial plantea dos puntos trascendentes: cambiar la mirada biomédica, para atender al otro respetándolo como persona, mirándolo, como dice Levinas: "La visión no es una trascendencia, otorga una significación por la relación que hace posible" (8); y ayudarse de la tecnología para otorgar cuidados y no de tecnologizarlos.

- En la formación, la importancia de impregnar a los estudiantes en el cuidar con empatía, con respeto, independiente del diagnóstico y de los modelos de atención que tenga para ello. Además, al formar en investigación inculcar en los futuros profesionales la relevancia de la investigación para la disciplina, ya que ésta encausará sus acciones y saberes. Sin embargo, para la realización de éstas se deben considerar principios éticos y el respeto a la persona humana.

- Finalmente, queda la sensación de que la enfermería aún tiene mucho por hacer y mejorar, que el compromiso seguirá siendo arduo y trabajoso, pero la enfermería no ha sido fácil y todos los profesionales que forman esta disciplina tienen la fuerza suficiente para hacer de su quehacer algo importante, como dice Tolle: "Hay que tener claro 
que nuestro presente es el único tiempo real, debemos vivirlo", sin dejar de lado nuestro centro: la persona humana (14).

\section{REFERENCIAS}

1. Watson J. Ponencia del primer Congreso internacional de Enfermería. Facultad de Enfermería y Nutriología. Universidad Autónoma de Chihuahua. [acceso: 18 de junio 2009]. Disponible en: http:// fen.uach.mx/index.php?page/Semblanza_Jean_Watson. Comentado en: Revista Actualizaciones en Enfermería. 2007; 10(4): 15-21.

2. Walker CA. Coalescing the theories of two nurse visionaries: Parse and Watson. J Adv Nurs. 1996; 24(5): 988-996.

3. Guillaumet M, Fargues I, Subirana M, Bros M. Teoría del cuidado humano: un café con Watson. Metas de enfermería. 2005; 8(2): 28-32.

4. Norma General administrativa $N^{\circ} 19$ Gestión del cuidado de enfermería para la atención cerrada. Resolución 1127. Ministerio de Salud de Chile (14 de diciembre de 2007).

5. Álvarez O, Román de Cisneros G. Sensibilidad. Valor condicional del Equipo de Salud para el Cuidado Humano [Revista on Line] 2008 julio. Hallado en: http:// portalesmedicos.com/publicaciones/ articles/1187/1/Sencibilidad-Valor-condicional-del-Equipo-de-Salud-para-elCuidado-Humano.html [Acceso: 20 de julio 2009]

6. McFarland M. Teoría de la diversidad y de la universalidad de los cuidados culturales. Modelos y teorías en enfermería. $6^{\mathrm{a}}$ ed. Madrid: Elsevier Mosby; 2007. p.472498.

7. Poblete M, Valenzuela S. Cuidado Humanizado: un desafío para las enfermeras en los servicios hospitalarios. Acta Paul. Enferm. 2007; 20(4): 499-503.

8. Levinas E. Totalidad e infinito: Ensayo sobre la exterioridad. $3^{a}$ ed. España: Ediciones Sígueme; 2005.

9. Vila V, Rossi L. Significado cultural del cuidado humanizado en la unidad de terapia intensiva: Mucho se habla y poco se vive. Rev. lat.-am. enferm. 2002; 10(2): 137-144.

10. Rubio L, Cosi M, Martínez C, Miró A, Sans L. Relaciones interdisciplinarias y humanización en las unidades de cuidados intensivos. Enferm. intensiva. 2006; 17(4):141-153.

11. Furegato A, Prestupa S. O que a populaçao sabe e espera do enfermeiro. Rev. Gaúcha Enferm. 2000; 20(1):80-90. Comentado en: Ciencia y cuidado. 2007; 4(4):21-27.

12. Ferreira C. Enfermería y cuidados intensivos: una mirada a la luz de la bioética. Enferm. Actual. 2006; 8(1): 23-25.

13. Código deodontológico del Consejo internacional de enfermeras (CIE). Suiza: Fornara, 2006; 2-8.

14. Tolle E. El poder del ahora: un camino hacia la realización personal. $1^{\text {a }}$ ed. USA: Norma; 2008. 